УДК 517.5

MSC $65 \mathrm{C} 60$

\title{
EQUALITY OF LS AND AITKEN ESTIMATIONS OF THE HIGHER COEFFICIENT OF THE LINEAR REGRESSION MODEL IN THE CASE OF CORRELATED DEVIATIONS
}

\author{
MARTA SAVKINA
}

Institute of Mathematics of NASU, Kyiv, Ukraine, E-mail: marta@imath.kiev.ua

\section{РІВНІСТЬ ОЦІНОК МНК ТА ЕЙТКЕНА СТАРШОГО КОЕФІЦІЕНТУ ЛІНІЙНОЇ МОДЕЛІ РЕГРЕСЇ̈ У ВИПАДКУ КОРЕЛЬОВАНИХ ВІДХИЛЕНЬ}

\author{
M. Ю. САBKIHA
}

Інститут математики НАН України, Київ, Україна, E-mail: marta@imath.kiev.ua

Abstract. At the paper a linear regression model whose function has the form $f(x)=a x+b, a$ and $b$ - unknown parameters, is studied. Approximate values (observations) of functions $f(x)$ are registered at equidistant points $x_{0}, x_{1}, \ldots, x_{n}$ of a line segment. It is also assumed that the covariance matrix of deviations is the Toeplitz matrix. Among all Toeplitz matrices, a family of matrices is selected for which all diagonals parallel to the main, starting from the $(k+$ 1)th, are zero, $k=n / 2, n-$ even. Elements of the main diagonal are denoted by $\lambda$, elements of the $k$ th diagonal are denoted by $c$, elements of the $j$ th diagonal are denoted by $c_{k-j}, j=1,2, \ldots, k-1$. The theorem proved at the paper states that if $c_{j}=(k /(k+1))^{j} c$, $j=1,2, \ldots, k-1$, that the LS estimation and the Aitken estimation of the $a$ parameter of this model coincide for any values $\lambda$ and $c$, which provide the positive definiteness of the resulting matrix.

KEYWORDS: least square method, regression model, Aitken estimation.

АнотАція. В роботі вивчається регресійна модель, функція якої має вигляд $f(x)=a x+b$, де $a$ та $b-$ невідомі параметри. Також припускається, що коваріаційна матриця відхилень є матрицею Тепліца. Наближені значення (спостереження) функції $f(x)$ реєструються у рівновіддалених точках відрізку [0, 1]. В теоремі, яку доведено в роботі, у випадку непарної кількості точок спостереження знайдено певний вигляд матриці Тепліца, який забезпечує рівність оцінки MHK та оцінки Ейткена параметра $а$ даної моделі. При такому вигляді коваріаційної матриці відхилень оцінки Ейткена та МНК параметра $b$ не будуть збігатися.

КЛЮчові СловА: метод найменших квадратів, регресійна модель, оцінка Ейткена. 


\section{ВСТУП}

В класичній perpeciї передбачається, що відхилення в perpeciйній моделі гомоскедастичні та не корелюють одне з іншим. Це доволі жорстка умова, яка досить часто не виконується. В зв'язку з цим актуально дослідження моделі, в якій відхилення мають різну дисперсію та корелюють одне з іншим. Проте в такому випадку коваріаційна матриця відхилень вже не буде одиничною, й оцінка звичайного методу найменших квадратів (MHK) невідомих параметрів моделі втрачає свої оптимальні властивості. Точніше, вона залишається незміщеною та спроможною, але вже не буде ефективною.

У випадку корельованих відхилень з різними дисперсіями ефективну оцінку, яка називається оцінкою Ейткена, дає зважений МНК. В формулу оцінки Ейткена входить вигляд коваріаційної матриці відхилень, тобто використання цієї оцінки передбачає знання такої матриці. А вона на практиці як правило невідома. Тому доводиться користуватися оцінкою МНК.

Отже порівняння цих оцінок, встановлення зв'язку між ними, а також знаходження випадків, коли вони збігаються, має важливе значення.

В [1] у випадку моделі, лінійної по параметрам, доведено теорему, яка стверджує, що для збігу оцінки МНК та оцінки Ейткена необхідно і достатньо, щоб кожну незалежну змінну можно було представити у вигляді лінійної комбінації деяких характеристичних векторів коваріаційної матриці відхилень. Причому одних й тих самих характеристичних векторів; їх кількість має дорівнювати кількості незалежних змінних моделі.

Якщо оцінка МНК та оцінка Ейткена відрізняються одна від одної, досліджується задача, яку похибку ми допускаємо, коли використовуємо оцінку MHK замість оцінки Ейткена. В якості міри ефективності оцінки МНК беруть відношення визначників коваріаційних матриць цих оцінок.

В [1] доведено, що міра ефективності оцінки МНК завжди менше або дорівнюе одиниці; дорівнюе одиниці вона тоді і тільки тоді, коли оцінка MHK та оцінка Ейткена збігаються. Також отримано формулу для міри ефективності оцінки МНК.

Ватсон у своїх роботах [7-8] отримав границі знизу для міри ефективності оцінки МНК. Одна з цих границь є функцією відношення найменшого характеристичного числа коваріаційної матриці відхилень до найбільшого характеристичного числа цієї матриці. Інша границя залежить від усіх характеристичних чисел коваріаційної матриці.

Гренандер [5] та Розенблат [6] вивчали асимптотичну ефективність оцінки МHK.

В статті [9] у випадку гетероскедастичних незалежних відхилень вивчається лінійна регресійна модель, функція якої має вигляд

$$
f(x)=a x+b .
$$

Знайдено умови на дисперсії відхилень, при яких оцінка Ейткена збігається з оцінкою МНК окремо для кожного невідомого параметру моделі. При цих умовах оцінки Ейткена та MHK параметра іншого параметру не будуть збігатися. 
1. ОЦІНКА МНК ТА ОЦІНКА ЕЙТКЕНА ЛІНІЙНОЇ РЕГРЕСІЙНОЇ МОДЕЛІ У ВИПАДКУ ГЕТЕРОСКЕДАСТИЧНИХ НЕЗАЛЕЖНИХ ВІДХИЛЕНЬ

Розглянемо модель регресії

$$
y_{i}=a x_{i}+b+\epsilon_{i}, \quad i=0,1, \ldots, n,
$$

де $\epsilon_{0}, \ldots, \epsilon_{n}-$ випадкові величини з $E \epsilon_{i}=0$ та коваріаційною матрицею $\sigma^{2} \Omega_{c}, n-$ парне, а $\Omega_{c}$ - додатньо визначена матриця, що має вигляд

$\Omega_{c}=\left(\begin{array}{ccccccccccc}\lambda & c_{k-1} & \ldots & c_{2} & c_{1} & c & 0 & 0 & \ldots & 0 & 0 \\ c_{k-1} & \lambda & \ldots & c_{3} & c_{2} & c_{1} & c & 0 & \ldots & 0 & 0 \\ \cdot & \cdot & \ldots & \cdot & \cdot & \cdot & \cdot & . & \ldots & \cdot & . \\ c_{1} & c_{2} & \ldots & c_{k-1} & \lambda & c_{k-1} & c_{k-2} & c_{k-3} & \ldots & c & 0 \\ c & c_{1} & \ldots & c_{k-2} & c_{k-1} & \lambda & c_{k-1} & c_{k-2} & \ldots & c_{1} & c \\ 0 & c & \ldots & c_{k-3} & c_{k-2} & c_{k-1} & \lambda & c_{k-1} & \ldots & c_{2} & c_{1} \\ . & . & \ldots & . & . & \cdot & . & . & \ldots & . & \cdot \\ 0 & 0 & \ldots & 0 & c & c_{1} & c_{2} & c_{3} & \ldots & \lambda & c_{k-1} \\ 0 & 0 & \ldots & 0 & 0 & c & c_{1} & c_{2} & \ldots & c_{k-1} & \lambda\end{array}\right)$ $\lambda>0, \quad k=\frac{n}{2}$.

В [2] знайдено формули для оцінки МНК та оцінки Ейткена невідомих параметрів моделі лінійної регресії загального вигляду. 3 цих формул у випадку моделі (1) та $x_{i}=\frac{i}{n}, i=0,1, \ldots, n$, маємо такі оцінки МНК та Ейткена параметрів $a$ та $b$ :

$$
\begin{gathered}
\hat{a}_{M N K}=\frac{12 n}{(n+1)(n+2)} \sum_{i=0}^{n}\left(\frac{i}{n}-\frac{1}{2}\right) y_{i}, \\
\left(\begin{array}{c}
\hat{a}_{A I T} \\
\hat{b}_{A I T}
\end{array}\right)=\left(X^{\prime} \Omega_{c}^{-1} X\right)^{-1} X^{\prime} \Omega_{c}^{-1} \vec{y},
\end{gathered}
$$

де

$$
\begin{gathered}
X^{\prime}=\left(\begin{array}{cccccc}
0 & \frac{1}{n} & \frac{2}{n} & \ldots & \frac{n-1}{n} & 1 \\
1 & 1 & 1 & \ldots & 1 & 1
\end{array}\right), \\
\vec{y}^{\prime}=\left(y_{0}, y_{1}, \ldots, y_{n}\right) .
\end{gathered}
$$

2. УМОВИ НА КОВАРІАЦІЙНУ МАТРИЦЮ ВІДХИЛЕНЬ ДЛЯ ЗБІГУ ОЦННОК МНК ТА ЕЙТКЕНА ПАРАМЕТРУ $a$ ЛІНІЙНОЇ МОДЕЛІ

Має місце

Теорема 1. Якщо в моделі (1)

$$
c_{j}=\left(\frac{k}{k+1}\right)^{j} c, \quad j=1,2, \ldots, k-1,
$$

$\lambda$ тас - будь-які величини, які забезпечують додатню визначеність матричі $\Omega_{c}$, то оиінка Ейткена та оцінка MHK параметра а збігаються. 
Доведення. Матриця $X^{\prime} \Omega_{c}^{-1}$ має розмір $2 \times(n+1)$, позначимо $i$-й елемент першого та другого рядка через $a_{i}$ та $b_{i}, i=0,1, \ldots, n$, відповідно. Помітимо, що

$$
b_{i}=b_{n-i}, \quad i=0,1, \ldots, k-1 .
$$

В цих позначеннях маємо

$$
X^{\prime} \Omega_{c}^{-1} X=\left(\begin{array}{cc}
\frac{1}{2 k} \sum_{i=0}^{2 k} i a_{i} & \sum_{i=0}^{2 k} a_{i} \\
\sum_{i=0}^{k-1} b_{i}+\frac{1}{2} b_{k} & 2 \sum_{i=0}^{k-1} b_{i}+b_{k}
\end{array}\right) .
$$

Позначимо через $\Delta_{c}$ визначник матриці $X^{\prime} \Omega_{c}^{-1} X$. Отримуємо

$$
\Delta_{c}=\left(2 \sum_{i=0}^{k-1} b_{i}+b_{k}\right)\left(-\frac{1}{2 k} \sum_{i=0}^{k-1}(k-i) a_{i}+\frac{1}{2 k} \sum_{i=k+1}^{2 k}(i-k) a_{i}\right) .
$$

Далі,

$$
\begin{gathered}
\left(X^{\prime} \Omega_{c}^{-1} X\right)^{-1} X^{\prime} \Omega_{c}^{-1}= \\
=\Delta_{c}^{-1}\left(\begin{array}{cc}
2 \sum_{i=0}^{k-1} b_{i}+b_{k} & -\sum_{i=0}^{k-1} b_{i}+\frac{1}{2} b_{k} \\
-\sum_{i=0}^{2 k} a_{i} & \frac{1}{2 k} \sum_{i=0}^{2 k} i a_{i}
\end{array}\right)\left(\begin{array}{cccc}
a_{0} & a_{1} & \ldots & a_{2 k} \\
b_{0} & b_{1} & \ldots & b_{2 k}
\end{array}\right) .
\end{gathered}
$$

Позначимо через $\hat{a}_{A I T}^{(j)}, j=0,1, \ldots, n, j$-й елемент першого рядка матриці $\left(X^{\prime} \Omega_{c}^{-1} X\right)^{-1} X^{\prime} \Omega_{c}^{-1}$. Маємо

де

$$
\begin{gathered}
\hat{a}_{A I T}^{(j)}=\Delta_{c}^{-1}\left[\left(2 \sum_{i=0}^{k-1} b_{i}+b_{k}\right) a_{j}-\left(\sum_{i=0}^{k-1} b_{i}+\frac{1}{2} b_{k}\right) b_{j}\right]= \\
=\frac{Z_{j}}{Z}, \quad j=0,1, \ldots, 2 k
\end{gathered}
$$

$$
\begin{gathered}
Z_{j}=2 a_{j}-b_{j}, \\
Z=-\frac{1}{k} \sum_{i=0}^{k-1}(k-i) a_{i}+\frac{1}{k} \sum_{i=k+1}^{2 k}(i-k) a_{i} .
\end{gathered}
$$

Доведемо, що якщо виконується умова (3), то

де

$$
Z_{j}=\frac{k-j}{k^{2}} D_{k}(c)
$$

$$
D_{k}(c)=\left(\left(2\left(\frac{k}{k+1}\right)^{k-1}-\frac{k+1}{k}\right) c-\frac{\lambda}{k}\right)^{-1} .
$$

Помітимо, що $a_{j}$ та $b_{j}, j=0,1, \ldots, n, \epsilon$ розв'язками систем лінійних алгебраїчних рівнянь $з$ матрицею $\Omega_{c}$ та стовпцями вільних членів

$$
\vec{x}_{a}{ }^{\prime}=\left(0, \frac{1}{n}, \frac{2}{n}, \ldots, \frac{n-1}{n}, 1\right) \quad \text { та } \quad \vec{x}_{b}{ }^{\prime}=(1,1,1, \ldots, 1,1)^{\prime},
$$


відповідно. Отже,

$$
Z_{j}=2 \frac{\left\|\Omega_{c, j}^{(a)}\right\|}{\left\|\Omega_{c}\right\|}-\frac{\left\|\Omega_{c, j}^{(b)}\right\|}{\left\|\Omega_{c}\right\|}=\frac{\left\|\Omega_{c, j}^{(0)}\right\|}{\left\|\Omega_{c}\right\|}
$$

де $\left\|\Omega_{c}\right\|$ - визначник матриці $\Omega_{c},\left\|\Omega_{c, j}^{(a)}\right\|,\left\|\Omega_{c, j}^{(b)}\right\|$ та $\left\|\Omega_{c, j}^{(0)}\right\|, j=0,1, \ldots, n-$ визначники матриць, які отримані з матриці $\Omega_{c}$ заміною $j$-го стовпця на стовпці $\vec{x}_{a}{ }^{\prime}, \vec{x}_{b}{ }^{\prime}$ та

$$
\vec{x}_{0}^{\prime}=\left(-1,-\frac{k-1}{k}, \ldots,-\frac{1}{k}, 0, \frac{1}{k}, \ldots, \frac{k-1}{k}, 1\right),
$$

відповідно.

Розглянемо добуток $P_{i}(c)(i=0,1, \ldots, k-1) i$-го рядка матриці $\Omega_{c}$ на $\vec{x}_{0}$. Маємо

$$
\begin{gathered}
P_{0}(c)=-\lambda \cdot 1-\sum_{j=1}^{i-1} \frac{k-j}{k} c_{k-j} ; \\
P_{i}(c)=-\sum_{j=0}^{i-1} \frac{k-j}{k} c_{k-i+j}-\lambda \cdot \frac{k-i}{k}- \\
-\sum_{j=i+1}^{2 i} \frac{k-j}{k} c_{k+i-j}-\sum_{j=2 i+1}^{k+i-1} \frac{k-j}{k} c_{k+i-j}+c \frac{i}{k}= \\
=-\frac{2(k-i)}{k} \sum_{j=k-i}^{k-1} c_{j}-\lambda \frac{k-i}{k}-\sum_{j=1}^{k-i-1} \frac{j-i}{k} c_{j}+c \frac{i}{k}, i=1,2, \ldots, k-2 ; \\
P_{k-1}(c)=-\frac{2}{k} \sum_{j=1}^{k-1} c_{k-j}-\frac{\lambda}{k}+c \frac{k-1}{k} .
\end{gathered}
$$

Якщо $i=k-2, k-3, \ldots, 0$, то враховуючи (7) отримуємо

$$
\begin{aligned}
& P_{i}(c)=-\frac{2(k-i)}{k} \sum_{j=1}^{k-1} c_{j}+\left(\frac{2(k-i)}{k} \sum_{j=1}^{k-i-1} c_{j}-\sum_{j=1}^{k-i-1} \frac{j-i}{k} c_{j}\right)+ \\
&-\lambda \frac{k-i}{k}+c \frac{i}{k}=\left(-\frac{2(k-i)}{k} \sum_{j=1}^{k-1} c_{j}-\lambda \frac{k-i}{k}+c \frac{(k-1)(k-i)}{k}\right)- \\
&-c \frac{(k-1)(k-i)}{k}+\sum_{j=1}^{k-i-1} \frac{2 k-i-j}{k} c_{j}+c \frac{i}{k}= \\
&=(k-i) P_{k-1}(c)+\sum_{j=1}^{k-i-1} \frac{2 k-i-j}{k} c_{j}-c(k-i-1) .
\end{aligned}
$$

Доведемо, що при умові (3)

$$
\sum_{j=1}^{k-i-1} c_{j} \frac{2 k-i-j}{k}-c(k-i-1)=0, i=0,1, \ldots, k-2 .
$$


Справді, якщо представити $c_{j}=\alpha^{j} c$, то ліва частина $(9)-$ поліном $(k-i-1)$-го степеня відносно $\alpha$, його можна розкласти на добуток

$$
\sum_{j=1}^{k-i-1} \alpha^{j} \frac{2 k-i-j}{k}-(k-i-1)=\left(\frac{k+1}{k} \alpha-1\right) \sum_{j=0}^{k-i-2} \alpha^{j}(k-i-j-1) .
$$
(9).

Якщо $\alpha=\frac{k}{k+1}$, то $\sum_{j=1}^{k-i-1} \alpha^{j}(2 k-i-j)-(k-i-1) k=0$, звідки випливає

Таким чином, з (8) при умові (3) отримуємо

$$
P_{i}(c)=(k-i) P_{k-1}(c), \quad i=0,1, \ldots, k-2 .
$$

За допомогою аналогічних міркувань, враховуючи, що $P_{k+1}(c)==-P_{k-1}(c)$, отримуємо

$$
\begin{gathered}
P_{i}(c)=(i-k) P_{k+1}(c)= \\
=(k-i) P_{k-1}(c), \quad i=k+1, k+2, \ldots, 2 k .
\end{gathered}
$$

Далі, розглянемо однорідну систему лінійних алгебраїчних рівнянь з матрицею $\Omega_{c, j}^{+}$, яка утворена з матриці $\Omega_{c}$ додаванням до $i$-го елемента $j$-го стовпця доданку $-\frac{(k-i) k}{k-j} P_{k-1}(c), i=0,1, \ldots, 2 k .3(10)$ та (11) випливає, що при умові (3) ця система має ненульовий розв'язок, а це значить, що

$$
\left\|\Omega_{c, j}^{+}\right\|=0,
$$

де $\left\|\Omega_{c, j}^{+}\right\|$- визначник матриці $\Omega_{c, j}^{+}$.

Далі, оскільки

$$
\left\|\Omega_{c, j}^{+}\right\|=\left\|\Omega_{c}\right\|-\frac{k^{2}}{k-j} P_{k-1}(c)\left\|\Omega_{c, j}^{(0)}\right\|,
$$

з (12) та (13) отримуємо

$$
\left\|\Omega_{c, j}^{(0)}\right\|=\frac{k-j}{k^{2}} P_{k-1}^{-1}(c)\left\|\Omega_{c}\right\| .
$$

Підставимо (3) в (7); отримуємо, що при умові (3)

$$
P_{k-1}(c)=D_{k}^{-1}(c) .
$$

Співвідношення (6) має місце для будь-яких $c_{j}, j=1,2, \ldots, k-1$, значить, і для тих, які визначаються формулою (3).

Підставимо (15) в (14), а (14) в (6). Отримуємо (5).

Далі, доведемо, що $Z$ при умові (3)

$$
Z=-\frac{(k+1)(2 k+1)}{6 k^{2}} D_{k}(c), j=0,1, \ldots, 2 k .
$$

Помітимо, що $Z$ можна переписати у вигляді

$$
Z=\frac{1}{k} \sum_{i=0}^{k-1}(k-i)\left(a_{2 k-i}-a_{i}\right)
$$


де

$$
a_{2 k-i}-a_{i}=\frac{\left\|\Omega_{c, 2 k-i}^{(a)}\right\|}{\left\|\Omega_{c}\right\|}-\frac{\left\|\Omega_{c, i}^{(a)}\right\|}{\left\|\Omega_{c}\right\|} .
$$

Розкладемо в $(18)$ визначники $\left\|\Omega_{c, 2 k-i}^{(a)}\right\|$ та $\left\|\Omega_{c, i}^{(a)}\right\|$ по $(2 k-i)$-му та $i$-му стовпцям відповідно згідно з теоремою Лапласа [3]. Маємо

$$
a_{2 k-i}-a_{i}=\left\|\Omega_{c}\right\|^{-1}\left(\sum_{l=0}^{2 k} \frac{l}{2 k} A_{l, 2 k-i}-\sum_{l=0}^{2 k} \frac{l}{2 k} A_{l, i}\right),
$$

де $A_{l, 2 k-i}$ та $A_{l, i}$ алгебраїчні доповнення до елементів $\omega_{l, 2 k-i}$ та $\omega_{l, i}$ матриці $\Omega_{c}$ відповідно. Доведемо, що для матриці $\Omega_{c}$ має місце рівність

$$
A_{l, 2 k-i}=A_{2 k-l, i} \text {. }
$$

Позначимо через $\Omega_{c, \pi}$ матрицю, яка утворена з матриці $\Omega_{c}$ розворотом на 180 градусів, а через $\bar{\Omega}_{c}^{(i, j)}$ та $\bar{\Omega}_{c, \pi}^{(i, j)}$ матриці, які утворені з матриць $\Omega_{c}$ та $\Omega_{c, \pi}$ відповідно викресленням $i$-го рядка та $j$-го стовпця, а через $\left\|\bar{\Omega}_{c}^{(i, j)}\right\|$ та $\left\|\bar{\Omega}_{c, \pi}^{(i, j)}\right\|$ - визначники матриць $\bar{\Omega}_{c}^{(i, j)}$ та $\bar{\Omega}_{c, \pi}^{(i, j)}$ відповідно.

Оскільки матриця $\Omega_{c}$ симетрична відносно головної та побічної діагоналей, то при розвороті на 180 градусів вона перейде сама в себе, тобто $\Omega_{c}=\Omega_{c, \pi} .3$ цього випливає, що $\bar{\Omega}_{c}^{(n-j, n-i)}=\bar{\Omega}_{c, \pi}^{(n-j, n-i)}$. Враховуючи, що

$$
A_{n-j, n-i}=(-1)^{2 n-i-j}\left\|\bar{\Omega}_{c}^{(n-j, n-i)}\right\|, \quad A_{n-j, n-i}^{(\pi)}=(-1)^{2 n-i-j}\left\|\bar{\Omega}_{c, \pi}^{(n-j, n-i)}\right\|,
$$

де $A_{n-j, n-i}^{(\pi)}-$ алгебраїчне доповнення до елемента $\omega_{n-j, n-i}^{(\pi)}$ матриці $\Omega_{c, \pi}$, отримуємо

$$
A_{n-j, n-i}=A_{n-j, n-i}^{(\pi)} .
$$

3 іншого боку, матриця $\bar{\Omega}_{c}^{(i, j)}$ при розвороті на 180 градусів переходить в матрицю $\bar{\Omega}_{c, \pi}^{(n-j, n-i)}$. В [4] доведено, що при розвороті будь-якої матриці порядку $N$ на 90 градусів їі визначник множиться на $(-1)^{C_{N}^{2}}$, значить, при розвороті на 180 градусів - на $(-1)^{2 C_{N}^{2}}$. Оскільки $2 C_{N}^{2}=N(N-1)-$ парне число для будь-якого $N$, то при розвороті на 180 градусів визначник матриці не змінюється, тобто

$$
A_{n-j, n-i}^{(\pi)}=A_{i, j} .
$$

В силу (21) та (22) $A_{i, j}=A_{n-j, n-i}$, тобто маємо (20).

Таким чином, з (19) з урахуванням (20) отримуємо

$$
\begin{gathered}
a_{2 k-i}-a_{i}=\left\|\Omega_{c}\right\|^{-1}\left(\sum_{l=0}^{2 k} \frac{l}{2 k} A_{2 k-l, i}-\sum_{l=0}^{2 k} \frac{l}{2 k} A_{l, i}\right)= \\
=\left\|\Omega_{c}\right\|^{-1} \sum_{l=0}^{2 k}\left(\frac{2 k-l}{2 k}-\frac{l}{2 k}\right) A_{l, i}=\left\|\Omega_{c}\right\|^{-1} \sum_{l=0}^{2 k} \frac{k-l}{k} A_{l, i}= \\
=-\left\|\Omega_{c}\right\|^{-1}\left\|\Omega_{c, i}^{(0)}\right\| .
\end{gathered}
$$


Якщо $c_{j}, j=1,2, \ldots, k-1$, визначаються формулою (3), то з (23) з урахуванням (14) маємо

$$
a_{2 k-i}-a_{i}=-\frac{k-i}{k^{2}} D_{k}(c) .
$$

Підставимо (24) в (17). Отримуємо

$$
Z=-\frac{D_{k}(c)}{k^{3}} \sum_{i=1}^{k} i^{2},
$$

звідки випливає (16).

3 (4), (5) та (16) отримуємо

$$
\hat{a}_{A I T}^{(j)}=-\frac{6(k-j)}{(k+1)(2 k+1)}, \quad j=0,1, \ldots, 2 k .
$$

Далі, оцінку $\hat{a}_{A I T}$ можна подати у вигляді

$$
\hat{a}_{A I T}=\sum_{i=0}^{n} \hat{a}_{A I T}^{(j)} y_{j} .
$$

З урахуванням (16) вона збігається з (2).

\section{ЛIтеРАТУРА}

1. Anderson T. The statistical analysis of time series. Moscow: Mir, 1976. 756 p. (in Russian)

2. Demidenko E. Z. Linear and nonlinear regression. Moscow: Finance and Statistics, 1981. 304 p. (in Russian)

3. Kurosh A. G. Advanced Algebra Course. Moscow: Nauka, 1965. 431 p. (in Russian)

4. Proskuryakov E. Z. Collection of problems in linear algebra. Moscow: Nauka, 1970. 384 p. (in Russian)

5. Grenander U. On the estimation of the regression coefficients in the case of the autocorrelated disturbance. Ann. Math. Statist. 1954. 25. P. 252-272.

6. Rosenblatt M. Serial correlation in Some regression problems in the time series analysis. Proc. Third Berkelay Symposium on Mathematical Statistics and Probability (J. Neyman, ed.). Vol. 1. Univ. of California Press, Berkelay and Los Angeles. 1956. P. $165-186$.

7. Watson G. S. Serial correlation in regression analysis, I. Biometrika. 1955. 42. P. 327-341.

8. Watson G. S. Linear least squares regression. Ann. Math. Statist. 1967. 38. P. 16791699.

9. Savkina M. Conditions for the coincidence of the IS and Aitken estimations of the parameters of the linear regression model. Journal of Numerical and Applied Mathematics. 2018. No. 3 (129). P. 36-44. (in Ukrainian)

Надійшла: 25.10.2021 / Прийнята: 10.11.2021 
РАВЕНСТВО ОЦЕНОК МНК И ЭЙТКЕНА СТАРШЕГО КОЭФФИЦИЕНТА ЛИНЕЙНОЙ МОДЕЛИ РЕГРЕССИИ В СЛУЧАЕ КОРРЕЛИРОВАННЫХ ОТКЛОНЕНИЙ

\footnotetext{
М. Ю. САВКIHA

Институт математики НАН Украины, Киев, Украина, E-mail: marta@imath.kiev.ua АннотАция. В работе изучена регрессионная модель с функцией вида $f(x)=a x+b$, где $a$ и $b-$ неизвестные параметры. Предполагается, что ковариационная матрица отклонений является матрицей Теплица. Приближенные значения (наблюдения) функции $f(x)$ регистрируются в равноудаленных точек отрезка $[0,1]$. В доказанной теореме для случая нечетного количества точек наблюдения найдено вид матрицы Теплица, который обеспечивает равенство оценок MHК и оценки Эйткена параметра $a$ данной модели. При таком виде ковариационной матрицы отклонений оценки Эйткена и МНК параметра $b$ не будут совпадать.

КЛЮчЕВЫЕ сЛовА: метод наименьших квадратов, регрессионная модель, оценка Эйткена.
} 